\title{
Oral Solution in Single-dose Container
}

National Cancer Institute

\section{Source}

National Cancer Institute. Oral Solution in Single-dose Container. NCI Thesaurus. Code C149721.

Medicinal product consisting of an oral solution presented in a single-dose container. 